\title{
Comparison of human gut microbiota in control subjects and patients with colorectal carcinoma in adenoma: Terminal restriction fragment length polymorphism and next-generation sequencing analyses
}

\author{
CHIKA KASAI $^{1}$, KAZUSHI SUGIMOTO ${ }^{2,3}$, ISAO MORITANI ${ }^{1}$, JUNICHIRO TANAKA $^{1}$, YUMI OYA ${ }^{1}$, \\ HIDEKAZU INOUE ${ }^{1}$, MASAHIKO TAMEDA ${ }^{2,3}$, KATSUYA SHIRAKI ${ }^{3}$, \\ MASAAKI ITO $^{4}$, YOSHIYUKI TAKEI ${ }^{3}$ and KOJIRO TAKASE ${ }^{1}$ \\ ${ }^{1}$ Department of Gastroenterology, Mie Prefectural General Medical Center, Yokkaichi, Mie 510-8561; Departments of \\ ${ }^{2}$ Molecular and Laboratory Medicine and ${ }^{3}$ Gastroenterology and Hepatology, Mie University School of Medicine, \\ Tsu, Mie 514-8507; ${ }^{4}$ Department of Cardiology and Nephrology, Tsu, Mie, Japan
}

Received July 3, 2015; Accepted August 13, 2015

DOI: $10.3892 /$ or.2015.4398

\begin{abstract}
Colorectal cancer (CRC) is the third leading cause of cancer-related deaths in Japan. The etiology of CRC has been linked to numerous factors including genetic mutation, diet, life style, inflammation, and recently, the gut microbiota. However, CRC-associated gut microbiota is still largely unexamined. This study used terminal restriction fragment length polymorphism (T-RFLP) and next-generation sequencing (NGS) to analyze and compare gut microbiota of Japanese control subjects and Japanese patients with carcinoma in adenoma. Stool samples were collected from 49 control subjects, 50 patients with colon adenoma, and 9 patients with colorectal cancer (3/9 with invasive cancer and $6 / 9$ with carcinoma in adenoma) immediately before colonoscopy; DNA was extracted from each stool sample. Based on T-RFLP analysis, 12 subjects (six control and six carcinoma in adenoma subjects) were selected; their samples were used for NGS and species-level analysis. T-RFLP analysis showed no significant differences in bacterial population between control, adenoma and cancer groups. However, NGS revealed that i), control and carcinoma in adenoma subjects had different gut microbiota compositions, ii), one bacterial genus (Slackia)
\end{abstract}

Correspondence to: Dr Kazushi Sugimoto, Department of Molecular and Laboratory Medicine, Mie University School of Medicine, 2-174 Edobashi, Tsu, Mie 514-8507, Japan

E-mail:kazushi@clin.medic.mie-u.ac.jp

Abbreviations: T-RFLP, terminal restriction fragment length polymorphism; CRC, colorectal cancer; NGS, next-generation sequencing

Key words: colorectal cancer, microbiota, terminal restriction fragment length polymorphism, next-generation sequencing was significantly associated with the control group and four bacterial genera (Actinomyces, Atopobium, Fusobacterium, and Haemophilus) were significantly associated with the carcinoma-in-adenoma group, and iii), several bacterial species were significantly associated with each type (control: Eubacterium coprostanoligens; carcinoma in adenoma: Actinomyces odontolyticus, Bacteroides fragiles, Clostridium nexile, Fusobacterium varium, Haemophilus parainfluenzae, Prevotella stercorea, Streptococcus gordonii, and Veillonella dispar). Gut microbial properties differ between control subjects and carcinoma-in-adenoma patients in this Japanese population, suggesting that gut microbiota is related to CRC prevention and development.

\section{Introduction}

Colorectal cancer (CRC), the third leading cause of cancer-related deaths in Japan, has an etiology linked to numerous factors including genetic mutation, diet, life style, and inflammatory process. The human gut is continually colonized by complex microbial communities in which the combined number of cells $\left(10^{11-13}\right.$ cells/g in the colon) is greater than the total number of the host cells (1). Therefore, the human body harbors 10 times more exogenous cells than human cells. The human gut microbiota becomes relatively stable around 1 week after birth, begins to resemble that of an adult after weaning, and once established remains stable over lifetime (2). It is generally believed that each healthy individual has his or her own unique gut microbiota $(3,4)$.

Numerous researchers have catalogued the gut microbiota of healthy humans and the gut microbiota associated with inflammatory bowel disease (IBD) (5-7) or obesity (8-10). In addition, a recent study suggests that the gut microbiota is associated with CRC development. Several plausible mechanisms in which the gut microbiota could interface with CRC have been proposed; for example, inflammation, DNA-damaging 
effects, and non-DNA-damaging effects could each be mechanistically important (11).

There is a considerable amount of research confirming that the gut microbiota is a primary driver of inflammation in the colon and the inflammatory environment is related to CRC development (12). Microbial dysbiosis (i.e., disturbance of the normal microbial community) can increase the proportion of facultative anaerobic bacteria, which include potentially harmful inflammation-inducing microorganisms. Inflammation driven by such bacteria (e.g., Bacteroides fragilis and Streptococcus bovis) is thought to affect carcinogenesis because these bacteria can activate immune cells to release promitogenic and proangiogenic cytokines such as IL-6 and IL-17 (13-15). In fact, there is epidemiological data that suggest up to $15 \%$ of human cancer incidence is inflammation-associated $(16,17)$.

DNA-damaging effects of microbiota in CRC are thought to be induced by microbes that produce numerous genotoxic substances and are thus linked to CRC development. For example, microbial-derived nitric oxide has the capacity to damage DNA $(18,19)$. Reactive oxygen species (ROS) are also powerful instigators of mutation and could contribute to chromosomal instability and risk of CRC $(20,21)$.

Carcinogenic effects of CRC-associated microbiota, effects that are unrelated to DNA damage, may be attributable to a number of bacterial metabolites. For example, hydrogen sulfide $\left(\mathrm{H}_{2} \mathrm{~S}\right)$ has been linked to $\mathrm{CRC}$ as a potential tumor-promoting agent. $\mathrm{H}_{2} \mathrm{~S}$ is produced by sulfate-reducing commensal bacteria as part of their normal metabolism (22). Although $\mathrm{H}_{2} \mathrm{~S}$ does not act as a direct DNA damaging agent, it modulates proliferation, apoptosis, and differentiation of colonic epithelial cells (23). Moreover, the gut microbiota metabolizes different dietary components to influence CRC development. For example, the gut microbiota metabolizes proteins from red meat to nitrosamine and heterocycle amines, and these metabolites are risk factors for CRC development $(24,25)$. In contrast, a high intake of dietary fiber has been considered to be protective against CRC development $(26,27)$; nevertheless, the beneficial effect of microbial fermentation of fiber and production of butyrate on CRC development is still an area of substantial controversy.

The gut microbiota and its products are clearly linked to CRC. However, most research has focused on the association between the gut microbiota and advanced CRC, rather than early-stage cancer. Thus, it is not clear whether the gut microbiota plays a role at an early stage of colorectal carcinogenesis. In addition, most studies in this field have been carried out in Western countries, and it is unknown whether certain members of the gut microbiota particularly associated with CRC also exist in the Japanese population, whose dietary habits and lifestyles are different from those of Western populations. In this study, we used next-generation sequencing (NGS) subsequent to terminal restriction fragment length polymorphism (T-RFLP) analysis to investigate the human gut microbiota in a Japanese population. We specifically selected patients with carcinoma in adenoma for the NGS analysis to evaluate possible associations of gut microbiota with early-stage cancer. We identified several potential bacterial genera and species uniquely associated with control specimens or with carcinoma-in-adenoma specimens.

\section{Materials and methods}

Human subjects. Subjects who were under 65 years of age and had undergone colonoscopy at the Mie Prefectural General Medical Center, Yokkaichi, Japan, between 2012 and 2013 were enrolled in the study. To evaluate differences in gut microflora via T-RFLP analysis, the subjects were classified into three groups as follows: i), control subjects, who had normal colonoscopy; ii), adenoma patients, who were diagnosed with colon adenoma bases on the colonoscopy; and iii), cancer patients, who had recently been diagnosed with CRC. Exclusion criteria for all participants included current use of antibiotics, history of or current chronic bowel or liver disease, history of chemotherapy or radiation therapy, and regular use of immunomodulators (steroids, interferons, etc.) or probiotics. Assignment of the patients is shown in Fig. 1. All patients received an explanation of the procedures and possible risks associated with the study, and they gave their written informed consent to participate. This study was performed in accordance with the Declaration of Helsinki and was approved by our Institutional Ethics Committee (authorized no. 2011-5; Mie Prefectural General Medical Center, Yokkaichi, Japan). Stool samples were collected from each participant prior to polyethylene-glycol preparation of the bowel for colonoscopy; each sample was stored at $4^{\circ} \mathrm{C}$ and submitted to Technosuruga Laboratory (Shizuoka, Japan) for the T-RFLP analysis, which is described below.

DNA extraction. Fecal samples ( $4 \mathrm{mg}$ each) were suspended in a solution containing $100 \mathrm{mM}$ Tris-HCI, pH 9.0, $40 \mathrm{mM}$ Tris-EDTA, $\mathrm{pH} 8.0$, and $4 \mathrm{M}$ guanidine thiocyanate. A $0.8-\mathrm{ml}$ aliquot of each suspension was homogenized with zirconia beads in a 2.0-ml screw cap tube with a FastPrep 24 Instrument (MP Biomedicals, Santa Ana, CA, USA) run at $5 \mathrm{~m} / \mathrm{sec}$ for $2 \mathrm{~min}$ and placed on ice for $5 \mathrm{~min}$. Each sample was spun at 5,000 $\mathrm{x} g$ for $1 \mathrm{~min}$; an automatic nucleic acid extractor (Precision System Science, Chiba, Japan) was then used to extract DNA from a $200-\mu 1$ aliquot of each sample. MagDEA DNA 200 (GC; Precision System Science) was used as the reagent for automated nucleic acid extraction.

$T-R F L P$. The $16 \mathrm{~S}$ rDNA was amplified from human fecal DNA using the fluorescent-labeled 516f primer (5'-TGCCAGC AGCCGCGGTA-3'; Escherichia coli positions 516-532) and 1510r primer (5'-GGTTACCTTGTTACGACTT-3'; E. coli positions $1,510-1,492)$. HotStarTaq DNA polymerase by Gene Amp PCR system 9600 (Applied Biosystems, Foster City, CA, USA) was used for each amplification reaction. The amplification program was as follows: preheating at $95^{\circ} \mathrm{C}$ for $15 \mathrm{~min}$ and then 30 cycles of i), denaturation at $95^{\circ} \mathrm{C}$ for $30 \mathrm{sec}$, ii), annealing at $50^{\circ} \mathrm{C}$ for $30 \mathrm{sec}$, and iii), extension at $72^{\circ} \mathrm{C}$ for $1 \mathrm{~min}$, and finally, a terminal extension at $72^{\circ} \mathrm{C}$ for $10 \mathrm{~min}$. Amplified DNA was purified by a MultiScreen PCR96 Filter Plate (Millipore, Billerica, MA, USA) and verified by electrophoresis. The restriction enzymes were selected according to Nagashima et al $(28,29)$. In brief, 16S-rDNA PCR products were purified and digested with $10 \mathrm{U}$ of $B s l \mathrm{I}$ (New England BioLabs, Ipswich, MA, USA) at $55^{\circ} \mathrm{C}$ for $3 \mathrm{~h}$. An ABI PRISM $3130 x 1$ genetic analyzer was used to analyze the resultant DNA fragments, namely fluorescent-labeled terminal restriction 
fragments (T-RFs), and GeneMapper software (Applied Biosystems) was used to determine T-RF length and peak area for each sample. T-RFs were divided into 29 operational taxonomic units (OTUs). The OTUs were quantified as the percentage of individual OTU per total OTU area, which were expressed as the percentage of the area under the curve (\% AUC). The reference database, Human Fecal Microbiota T-RFLP profiling (http://www.tecsrg-lab.jp/), was used to putatively identify the bacteria in each classification unit and the corresponding OTU.

To evaluate differences in gut microbiota composition at the species level, samples from six control subjects and six patients with carcinoma in adenoma were selected for NGS; IBM SPSS software ver. 22 was used to match control and patient samples based on age, gender, and BMI. Subjects with carcinoma in adenoma were selected and subjects with advanced cancer were excluded to avoid the possibility of gut microbial environment alterations due to cancer progression.

Illumina library generation. NGS analysis of microbial community structure in each feces sample was performed with MiSeq (Illumina, San Diego, CA, USA), as previously described by Takahashi et al (30). Briefly, the V3-V4 region of 16S rDNA was amplified using 341F (5'-CCTACGGGAGG CAGCAG-3') (31) and 806R (5'-GGACTACHVGGGTWTCT AAT-3') (32) primers. In addition to the V3-V4-specific priming regions, these primers were complementary to standard Illumina forward and reverse primers. The reverse primer also contained a 6-bp indexing sequence (CAGATC, ACTTGA, GATCAG, TAGCTT, GGCTAC, CTTGTA, ATCACG, CGATGT, TTAGGC, TGACCA, ACAGTG and GCCAAT) to allow for multiplexing. The touchdown PCR method was used with a GeneAmp PCR system 9700 (Applied Biosystems) for thermal cycling. Each PCR reaction mixture $(25 \mu \mathrm{l})$ contained 20 ng genomic DNA, 2X MightyAmp Buffer ver. 2 (Takara), $0.25 \mu \mathrm{M}$ of each primer, and 1.25 units of MightyAmp DNA Polymerase (Takara). Each PCR amplification and preparation of amplicon pool were performed as described by Takahashi et al (30).

Illumina sequencing and quality filtering. As recommended by Illumina for the pooling of two libraries and described by Takahashi et al (30), each multiplexed library pool was spiked with $30 \%$ PhiX control to improve base calling during sequencing. Sequencing was conducted using a paired-end, 2x251-bp cycle run on an Illumina MiSeq sequencing system and MiSeq reagent Nano kit version 2 (500 cycle) chemistry. Paired-end sequencing with read lengths of $251 \mathrm{bp}$ was performed. After demultiplexing, a clear overlap in the paired-end reads was observed. This overlap allowed paired reads to be joined together with the fastq-join program (http://code.google.com/p/ea-utils/). Only reads that had quality value (QV) scores of $\geq 20$ for $>99 \%$ of the sequence were extracted for further analysis. All sequences with ambiguous base calls were discarded (30).

Bioinformatics analysis. Metagenome@Kin software (World Fusion Co., Ltd., Tokyo, Japan) was used to conduct homology searches of the TechnoSuruga Lab Microbial Identification Database DB-BA9.0 (TechnoSuruga Laboratory Co., Ltd.,

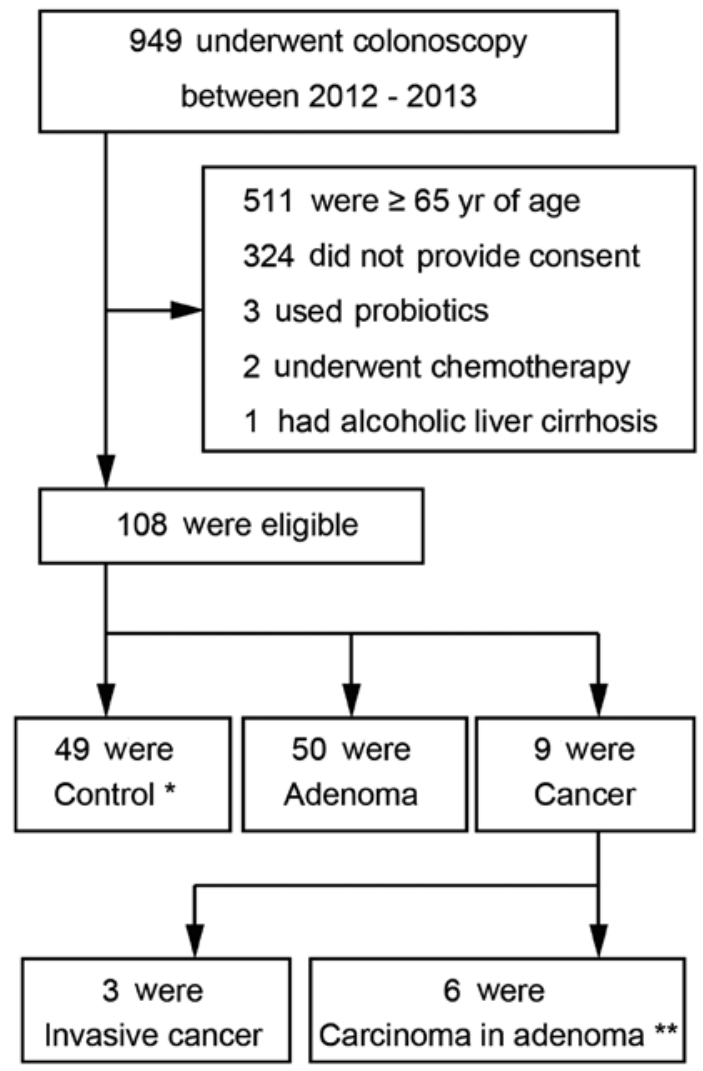

Figure 1. Flowchart showing the total number of participants enrolled and the final number of participants included in the study. Forty-nine control, 50 adenoma, and 9 cancer subjects (3/9 invasive cancer and 6/9 carcinoma in adenoma) were included in the study. * control and ${ }^{* *}$ used for NGS.

Shizuoka, Japan), which contains only bacteria with standing in the taxonomic nomenclature, with the $16 \mathrm{~S}$ rDNA sequences.

Statistical analysis. Data were analyzed using the Kruskal-Wallis test or the Mann-Whitney test (two-sided) for continuous variables and Fisher's exact test for categorical variables using IBM SPSS software ver. 22. P-values $<0.05$ were considered significant.

\section{Results}

Differences in bacterial community profiles between control, adenoma, and cancer subjects as determined by T-RFLP analysis. Demographic and clinical characteristics of the subject groups are shown in Table I. A total of 49 control subjects, 50 adenoma subjects, and 9 CRC subjects (3 with invasive cancer and 6 with carcinoma in adenoma) were enrolled in this study. Blood test results showed that total cholesterol and high-density-lipoprotein cholesterol levels were significantly lower in the cancer subjects. The average age and body mass index of the cancer subjects were each higher than those of the control and adenoma subjects. Differences in bacterial flora between the three groups are summarized in Table II. There were no significant differences in bacterial composition between each pair of groups.

Differences in bacterial communities between control and carcinoma in adenoma subjects by $16 \mathrm{~S}$ rRNA sequencing. 
Table I. Demographic and clinical characteristics of the study groups.

\begin{tabular}{|c|c|c|c|c|}
\hline & Control $(n=49)$ & Adenoma $(n=50)$ & Cancer $(n=9)^{b}$ & P-value \\
\hline Age (years) ${ }^{\mathrm{a}}$ & $48.8 \pm 8.2$ & $53.5 \pm 9.3$ & $54.3 \pm 7.9$ & 0.011 \\
\hline Gender, male; n (\%) & $21(42.9)$ & $28(56)$ & $4(44.4)$ & 0.399 \\
\hline BMI $\left(\mathrm{kg} / \mathrm{m}^{2}\right)^{\mathrm{a}}$ & $22.5 \pm 3.7$ & $24.2 \pm 3.9$ & $24.4 \pm 2.8$ & 0.030 \\
\hline Constipation; yes, n (\%) & $11(22.4)$ & $8(16.0)$ & $5(55.6)$ & 0.042 \\
\hline Alcohol intake; yes, $\mathrm{n}(\%)$ & $23(48.9)$ & $26(53.1)$ & $3(33.3)$ & 0.646 \\
\hline Smoking; yes, n (\%) & $9(18.8)$ & $13(26.5)$ & $3(33.3)$ & 0.480 \\
\hline \multicolumn{5}{|l|}{ Laboratory data } \\
\hline HbA1c (JDS; \%) & $5.4 \pm 0.8$ & $5.4 \pm 0.6$ & $5.4 \pm 0.7$ & 0.219 \\
\hline Total cholesterol (mg/dl) & $206.2 \pm 34.7$ & $195.2 \pm 32.1$ & $174.6 \pm 39.5$ & 0.021 \\
\hline Triglyceride (mg/dl) & $113.9 \pm 85.5$ & $128.7 \pm 80.0$ & $135.0 \pm 104.0$ & 0.229 \\
\hline HDL-cholesterol (mg/dl) & $70.7 \pm 22.1$ & $64.0 \pm 16.9$ & $52.1 \pm 14.5$ & 0.028 \\
\hline
\end{tabular}

${ }^{\mathrm{a}}$ Mean $\pm \mathrm{SD}$; bincludes invasive cancer $(\mathrm{n}=3)$, carcinoma in adenoma $(\mathrm{n}=6)$; $\mathrm{p}$-values are based on Kruskal-Wallis test for continuous variables and Fisher's exact test for categorical variable; BMI, body mass index; HDL, high-density lipoprotein.

Table II. Differences in bacterial flora based on T-RFLP analysis.

\begin{tabular}{lcccc}
\hline & Control & Adenoma & Cancer & P-value \\
\hline Bifidobacterium & $7.8 \pm 7.6$ & $8.1 \pm 7.4$ & $5.6 \pm 5.5$ & 0.838 \\
Lactobacillales & $5.7 \pm 8.1$ & $6.3 \pm 8.9$ & $2.3 \pm 2.2$ & 0.516 \\
$\begin{array}{l}\text { Bacteroides } \\
\text { Prevotella }\end{array}$ & $20.1 \pm 12.9$ & $37.5 \pm 15.0$ & $39.1 \pm 7.0$ & 0.835 \\
$\begin{array}{l}\text { Clostridium } \\
\text { cluster IV }\end{array}$ & $8.0 \pm 5.4$ & $2.7 \pm 7.2$ & $0.6 \pm 1.1$ & 0.637 \\
$\begin{array}{l}\text { Clostridium } \\
\text { subcluster XIVa }\end{array}$ & $21.5 \pm 7.9$ & $21.6 \pm 7.5$ & $22.4 \pm 10.2$ & 0.979 \\
$\begin{array}{l}\text { Clostridium } \\
\text { cluster XI }\end{array}$ & $2.0 \pm 4.0$ & $1.4 \pm 3.0$ & $2.9 \pm 2.5$ & 0.144 \\
$\begin{array}{l}\text { Clostridium } \\
\text { cluster XVIII }\end{array}$ & $1.7 \pm 2.4$ & $2.0 \pm 1.8$ & $1.8 \pm 2.8$ & 0.215 \\
\hline P-vat & & & & \\
\hline
\end{tabular}

P-values are based on Kruskal-Wallis test; data are expressed as mean $\pm \mathrm{SD}$; TRFLP, terminal restriction fragment length polymorphism.

Our T-RFLP analysis showed no significant differences in bacterial population between the control, adenoma, and cancer groups. However, in order to determine the possible presence of bacteria correlated with health and cancer, we selected 12 subjects (six control and six carcinoma in adenoma) from the initial groups for NGS (Table III). Using our primer set and MiSeq platform combination, an average of 24,084 reads were obtained for each sequencing reaction. Fig. 2 shows the phylotype distribution for individual subjects in this study. The composition and relative abundance of the major bacterial phyla were similar, with Bacteroidetes and Firmicutes being the dominant phyla. However, after dividing the samples into two groups (control vs. carcinoma in adenoma) and performing statistical analyses, a significant increase in the proportion of
Fusobacteria (control 0\% vs. carcinoma in adenoma 4\%) was observed in the carcinoma-in-adenoma group relative to the control group (Fig. 3). There were no between-group differences with regard to other bacteria.

Comparison of microbiomes at the genus level. Genus-level analyses identified one bacterial genus that was significantly associated with the control group (Slackia), and four bacterial genera were significantly associated with the carcinoma-in-adenoma group (Actinomyces, Atopobium, Fusobacterium, and Heamophilus) (Table IV).

Comparison of microbiomes at the species level. Species-level analyses identified one bacterial species (Eubacterium coprostanoligens) that was significantly associated with the control group and eight bacterial species (Actinomyces odontolyticus, Bacteroides fragilis, Clostridium nexile, Fusobacterium varium, Heamophilus parainfluenzae, Prevotella stercorea, Streptococcus gordonii, and Veillonella dispar) that were significantly associated with the carcinoma-in-adenoma group (Table V).

Most notably, the proportions of Actinomyces odontolyticus, Bacteriodes fragilis, and Heamophilus parainfluenzae were significantly higher in feces from carcinoma-in-adenoma subjects than in those from control subjects; in fact, these bacteria were barely detectable in feces from control subjects (Fig. 4A-C). In contrast, the proportions of Eubacterium coprostanoligens were significantly higher in feces from control subjects than in those from carcinoma-in-adenoma subjects; this bacteria was barely detectable in feces of carcinoma-in-adenoma subjects (Fig. 4D).

Although the genus Slackia was significantly associated with control subjects (Fig. 5A), there were no statistically significant between-group differences in the relative proportion of each individual Slackia species. Slackia species as a whole, however, were more abundant in feces from control subjects compared with that from carcinoma-in-adenoma subjects (Fig. 5B). 
Table III. Characteristics of the study subjects participating in next-generation sequencing analysis.

\begin{tabular}{|c|c|c|c|c|c|c|}
\hline Participant ID & Health status & Gender & Age (years) & BMI & Tumor size (mm) & Tumor location \\
\hline N1 & Healthy & M & 46 & 25.1 & & \\
\hline N2 & Healthy & M & 39 & 19.6 & & \\
\hline N3 & Healthy & $\mathrm{F}$ & 55 & 24.7 & & \\
\hline N4 & Healthy & $\mathrm{F}$ & 49 & 20.3 & & \\
\hline N5 & Healthy & M & 56 & 25.63 & & \\
\hline N6 & Healthy & M & 57 & 23.15 & & \\
\hline $\mathrm{C} 1$ & Cancer & M & 48 & 24.5 & 10 & Sigmoid \\
\hline $\mathrm{C} 2$ & Cancer & $\mathrm{F}$ & 64 & 26.9 & 20 & Sigmoid \\
\hline $\mathrm{C} 3$ & Cancer & M & 40 & 20.5 & 13 & Sigmoid \\
\hline $\mathrm{C} 4$ & Cancer & M & 62 & 24.9 & 20 & Sigmoid \\
\hline C5 & Cancer & M & 61 & 24.3 & 15 & Sigmoid \\
\hline C6 & Cancer & $\mathrm{F}$ & 49 & 19.46 & 10 & Descending \\
\hline
\end{tabular}

BMI, body mass index; F, female; M, male.

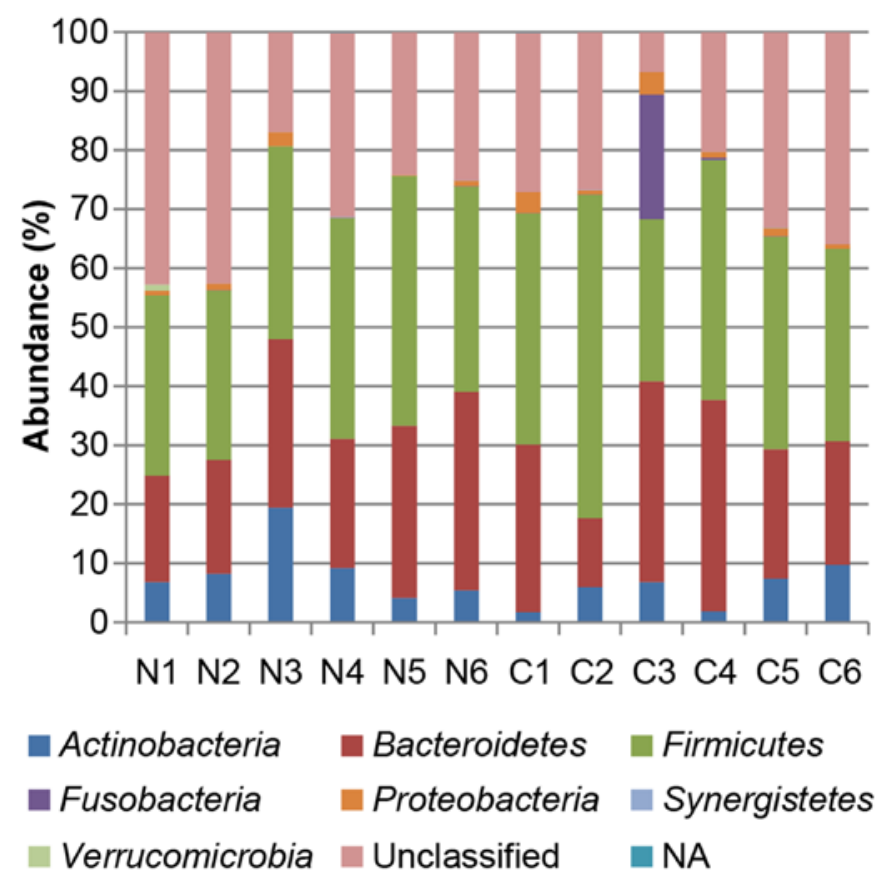

Figure 2. Phylum-level classification of bacteria identified in individual stool samples. N-numbered samples were obtained from normal control subjects, whereas $\mathrm{C}$-numbered samples were obtained from carcinoma-in-adenoma subjects. Each bar represents the percent contribution of phylum-level profiles grouped by N-C status or for each individual. The phyla represented by each color code are shown below the figure. NA, not assigned.

\section{Discussion}

Using NGS, we found that the gut microbiota differs between control and carcinoma-in-adenoma subjects; however, our initial T-RFLP analysis did not reveal any statistically significant differences in relative proportions of bacterial flora between control, adenoma, and carcinoma-in-adenoma subjects. We identified several potential gut microbial members significantly associated with the control and carcinoma-in-adenoma groups.
Table IV. Bacterial genera with significantly different groupspecific representation.

\begin{tabular}{lccc}
\hline & Ave. N (\%) & Ave. C (\%) & P-value \\
\hline Actinomyces & 0.022 & 0.116 & 0.037 \\
Atopobium & $\mathrm{ND}$ & 0.005 & 0.022 \\
Fusobacterium & 0.004 & 3.84 & 0.004 \\
Heamophilus & 0.002 & 0.027 & 0.020 \\
Slackia & 0.162 & 0.009 & 0.049
\end{tabular}

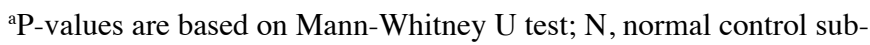
jects; C, carcinoma-in-adenoma patients; ND, not determined.

Phylum-level analyses revealed that the relative proportion of Fusobacterium was significantly higher in carcinoma-in-adenoma subjects than in control subjects. Fusobacterium has been studied recently because of its correlations with CRC $(33,34)$. There are two studies $(35,36)$ that investigated the mechanisms by which Fusobacterium nucleatum in the gut could be associated with CRC. The first study was conducted by Kostic et al; it suggested that $F$. nucleatum induced a nuclear factor $-\kappa \mathrm{B}(\mathrm{NF}-\kappa \mathrm{B})$-driven proinflammatory response to promote CRC (35). The second study was by Rubinstein et al; it provided mechanistic insights, most notably that the actions of Fusobacterium spp. were presumably mediated via binding of FadA, a virulence factor expressed on the bacterial cell surface, to receptors on host epithelial cells; this FadA-receptor binding seemed to modify barrier function, increase inflammation through the modulation of the tumor microenvironment, and activate pro-oncogenic signals to promote CRC (36).

Genus-level and species-level analyses showed that the genus Slackia and the species Eubacterium coprostanoligens were present in significantly higher proportions in control subjects compared with carcinoma-in-adenoma subjects. Slackia is one of the few characterized equol-forming gut 

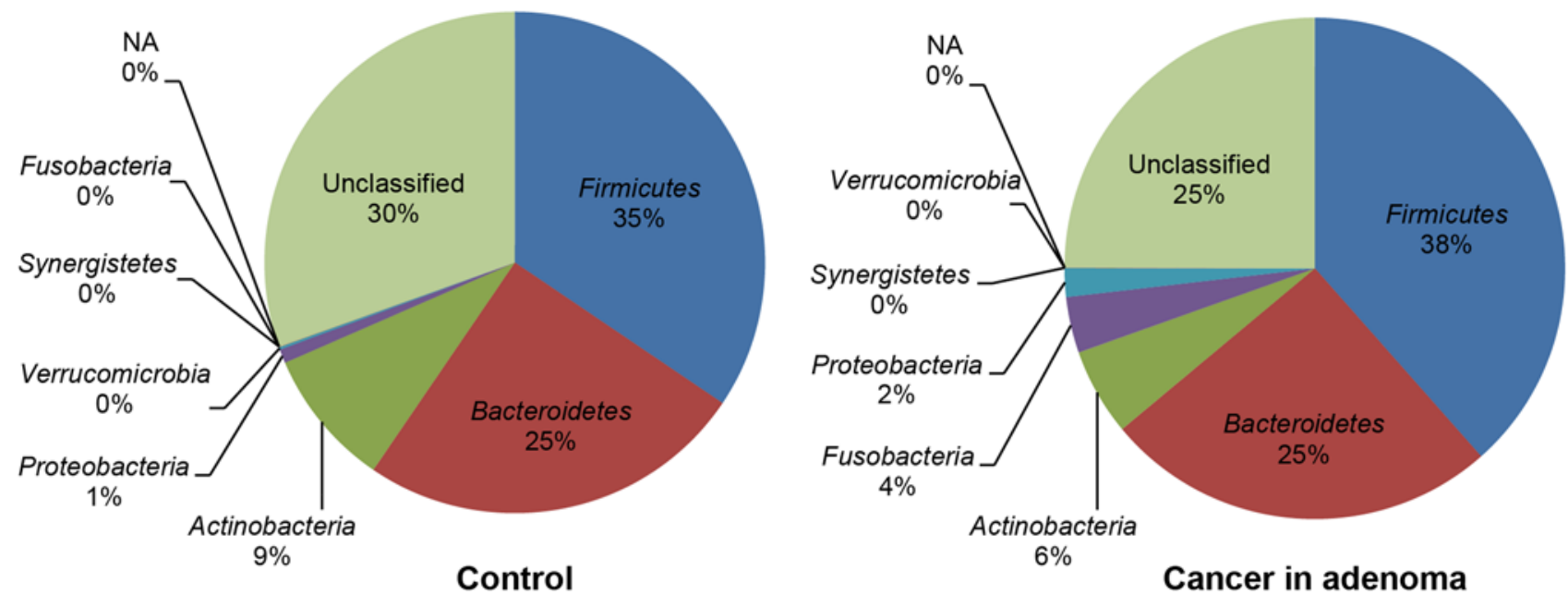

Figure 3. Average phylum distribution of gut microbiomes of control and carcinoma in adenoma subjects. NA, not assigned. ${ }^{*} \mathrm{P}<0.05$.

Table V. Bacterial species with significantly different groupspecific representation.

\begin{tabular}{|c|c|c|c|}
\hline & Ave. N (\%) & Ave. C (\%) & P-value \\
\hline $\begin{array}{l}\text { Actinomyces } \\
\text { odontolyticus }\end{array}$ & ND & 0.036 & 0.007 \\
\hline Bacteroides fragilis & 0.015 & 0.658 & 0.0046 \\
\hline Clostridium nexile & 0.067 & 0.661 & 0.036 \\
\hline $\begin{array}{l}\text { Eubacterium } \\
\text { coprostanoligens }\end{array}$ & 0.650 & ND & 0.022 \\
\hline Fusobacterium varium & ND & 0.268 & 0.022 \\
\hline $\begin{array}{l}\text { Heamophilus } \\
\text { parainfluenzae }\end{array}$ & 0.001 & 0.022 & 0.020 \\
\hline Prevotella stercorea & ND & 1.186 & 0.022 \\
\hline Streptococcus gordonii & 0.002 & 0.031 & 0.014 \\
\hline Veillonella dispar & 0.004 & 0.198 & 0.042 \\
\hline
\end{tabular}

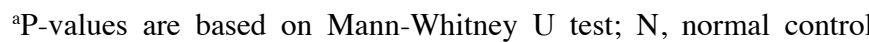
subject; C, carcinoma-in-adenoma patient; ND, not determined.

bacteria isolated from humans (37). Equol is produced from daidzein (a soy isoflavone) by intestinal bacteria in some, but not all, adults (38). An individual's capacity for equol production depends on the representation of equol-forming bacteria in the individual's intestine (39). Equol is produced in only $20-30 \%$ of adults who consume soy diets containing isoflavones in Western countries; in contrast, it is produced in no less than $50-60 \%$ of adults in Asian countries, and these adults more commonly consume soy diets (40-42). Soy isoflavones are often referred to as phytoestrogens; they have an estrogen-like chemical structure and can bind to estrogen receptors (43). Equol is the active form of soy isoflavones in the human intestine, and equol shows a stronger estrogen-like activity than daizein because it affects hormone-dependent diseases (44). Equol is anticipated to have a protective effect on prostate cancer development and to reduce the risk of mammary tumors $(45,46)$. We expect that the equol-forming bacteria Slackia have a preventive effect against CRC, as well.
Eubacterium, a beneficial genus of fecal bacteria, includes many species that produce butyrate $(47,48)$. Butyrate is regarded as the most important nutrient for epithelial cells of the colon, and it plays an essential role in the energy metabolism and normal development of these cells (49). Several studies have shown that butyrate is a beneficial inhibitor of colon carcinoma cell proliferation because it induces apoptosis in human colon carcinoma cells (50-52). Eubacterium coprostanoligens is a cholesterol-reducing bacterium (53). Cholesterol-reducing bacteria convert cholesterol to coprostanol, which is not absorbed by the human gastrointestinal system, thereby leading to reduced cholesterol levels. There is strong epidemiological evidence that links high fat consumption to increased risk of CRC $(54,55)$. We thus expect Eubacterium coprostanoligens to be another prospective inhibitor of CRC.

Genus-level analyses showed that four genera (Actinomyces, Atopobium, Fusobacterium, and Haemophilus) were present in significantly higher proportions in carcinomain-adenoma subjects than in control subjects. Species-level analyses showed that eight species (Actinomyces odontolyticus, Bacteroides fragilis, Clostridium nexile, Fusobacterium varium, Haemophilus parainfluenzae, Prevotella stercorea, Streptococcus gordonii, and Veillonella dispar) were present in significantly higher proportions in carcinoma-in-adenoma subjects than in control subjects. Here we focused on only three of these species (Actinomyces odontolyticus, Bacteroides fragilis, Haemophilus parainfluenzae sp); each was highly represented in carcinoma-in-adenoma subjects, but barely detected in control subjects.

Actinomyces odontolyticus are often present in the oral cavity and gastrointestinal tract of healthy humans. Some Actinomyces spp. are known to be opportunist pathogens associated with several colon-related diseases such as CRC and Crohn's disease. Bacteroides fragilis is a Gram-negative obligate anaerobe persistently preset in the colon of nearly all humans. It accounts for only $0.5 \%$ of the human gut microbiota; nevertheless, it has enterotoxigenicity and is considered to be pathogen important to CRC. Chronic inflammation may lead to the hypermethylation of DNA and drive cells to malignancy (56). Persistent enterotoxigenic Bacteroides 
A

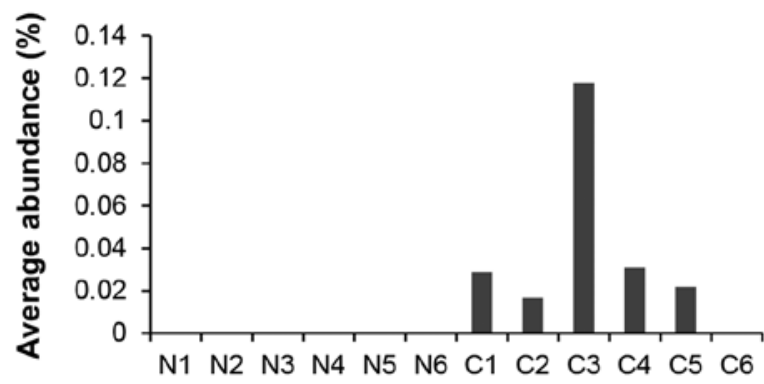

C
Haemophilus parainfluenzae

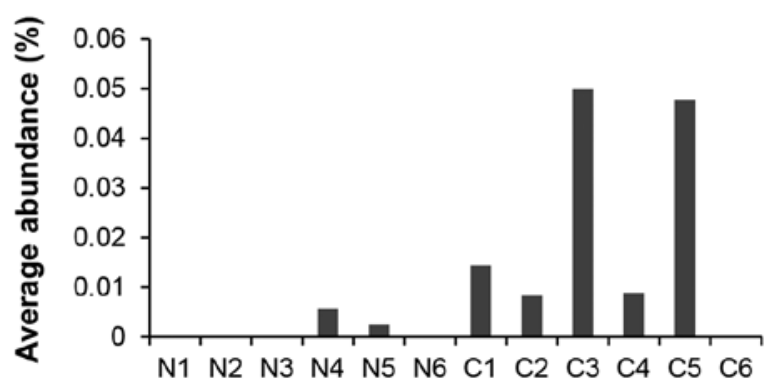

B

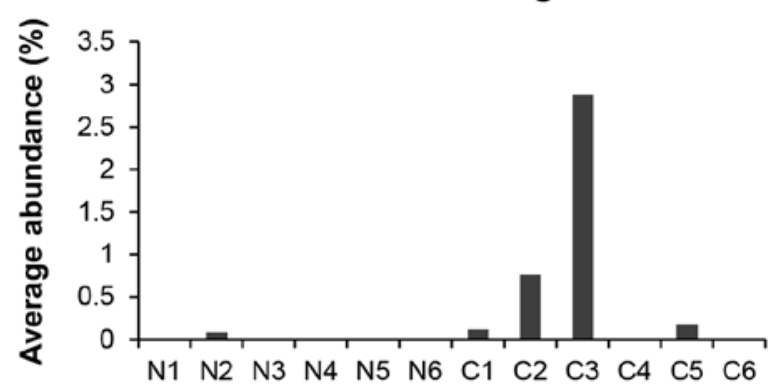

D Eubacterium coprostanoligenes

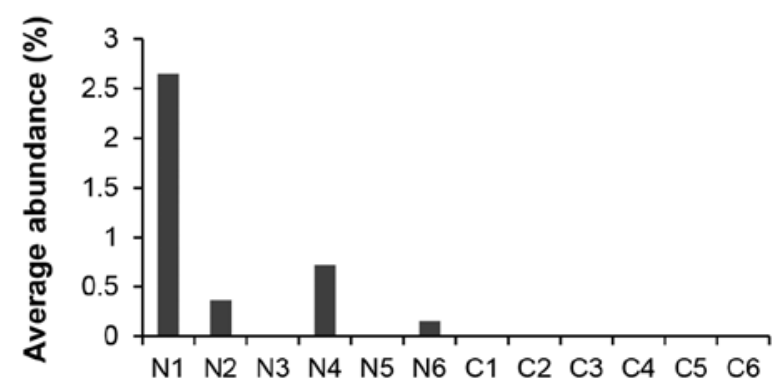

Figure 4. (A) Average abundance of Actinomyces odontolyticus in individual stool samples. N-numbered samples were obtained from normal control subjects and C-numbered samples were obtained from carcinoma-in-adenoma subjects. (B) Average abundance of Bacteroides fragilis in individual stool samples. (C) Average abundance of Haemophilus parainfluenzae in individual stool samples. (D) Average abundance of Eubacterium coprostanoligenes in individual stool samples.

A

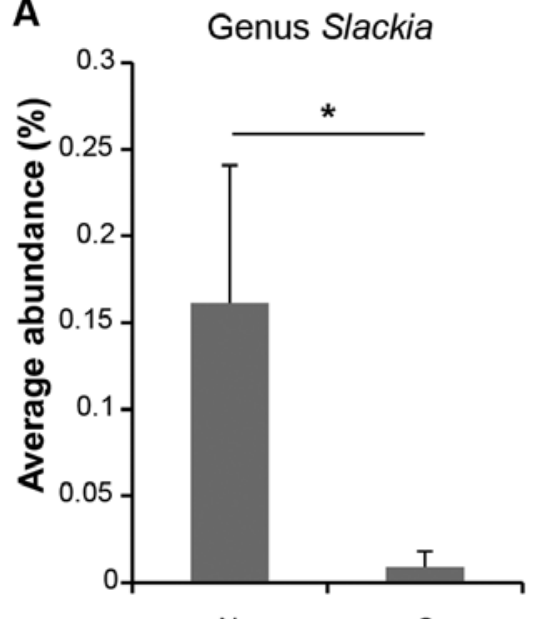

B

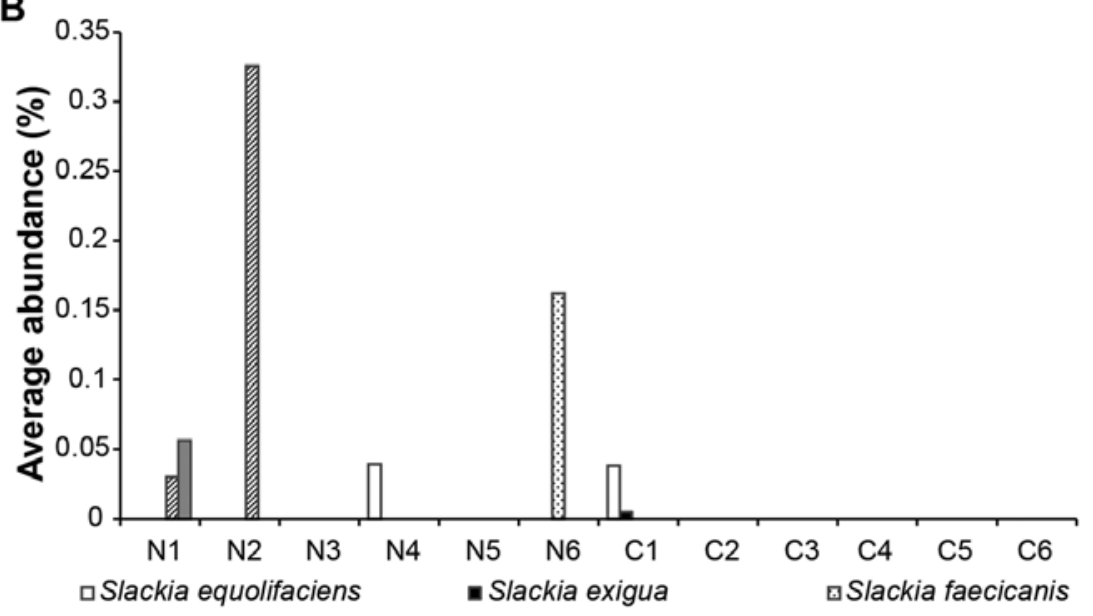

Figure 5. (A) Average abundance of the genus Slackia in individual stool samples. N-numbered samples were obtained from normal control subjects and $\mathrm{C}$-numbered samples were obtained from carcinoma-in-adenoma subjects. "P<0.05. (B) Average abundance of Slackia species in individual stool samples.

fragilis (ETBF) infection may increase the risk of colon carcinogenesis (57). Haemophilus parainfluenzae is a commensal species, which belongs to the phylum Proteobacteria. It is an opportunistic pathogen that may induce invasive infections such as pneumonia and endocarditis.

The control subjects in our study harbored beneficial bacterial species, whereas the carcinoma-in-adenoma subjects harbored harmful bacteria species that could act as opportunist pathogens and/or inflammation drivers. In the case of progressive CRC, the colonic environment can be modified by multiple factors such as epithelial cell apoptosis and cancer cachexia; therefore, it is often complicated whether the current microbial environment is a sequel of CRC or the gut microbiota is a driver of CRC development by way of inflammatory responses. However, the fact that the gut microbial profiles differed significantly even between control subjects and carcinoma-in-adenoma (i.e., relatively-early-stage cancer) subjects on the genus and species levels suggested that the microbial environment including the gut microbiota was an important etiologic factor for 
CRC. In order to determine the exact triggers of CRC, we should also carefully observe non-CRC patients harboring inflammation-driving microbes in a future long-term study of CRC progression.

We acknowledge this research was limited to the characterization of microbiota and that stool metabolites were not analyzed. We believe further related research that includes analysis of stool metabolites will definitely improve our understanding of the mechanisms that lead to CRC.

In conclusion, the results of the present study in a Japanese population showed that gut microbiota differed between control and carcinoma-in-adenoma subjects. In particular, the results suggested that the gut microbiota served as a driver of carcinogenesis because changes in the composition of the gut microbiota were observed even in carcinoma in adenoma, which is an early-stage cancer. However, further study will be necessary to clarify the precise mechanisms by which the gut microbiota drives carcinogenesis and to identify the cancer-associated microbial members. An improved understanding of mechanisms that cause gut microbiota metabolites to interface with carcinogenesis should lead to improved diagnostic, preventative, and therapeutic approaches; for example, probiotics may become useful as more natural and less disruptive treatments for the prevention of CRC and/or other GI-related disorders.

\section{Acknowledgements}

The authors appreciate TechnoSuruga Laboratory Co., Ltd. (Shizuoka, Japan) for technical assistance.

\section{References}

1. Savage DC: Microbial ecology of the gastrointestinal tract. Annu Rev Microbiol 31: 107-133, 1977.

2. Mitsuoka T and Hayakawa K: The fecal flora in man. I. Composition of the fecal flora of various age groups. Zentralbl Bakteriol Orig A 223: 333-342, 1973 (In German).

3. Zoetendal EG, Akkermans AD and De Vos WM: Temperature gradient gel electrophoresis analysis of $16 \mathrm{~S}$ rRNA from human fecal samples reveals stable and host-specific communities of active bacteria. Appl Environ Microbiol 64: 3854-3859, 1998.

4. Rajilić-Stojanović M, Heilig HG, Tims S, Zoetendal EG and de Vos WM: Long-term monitoring of the human intestinal microbiota composition. Environ Microbiol 15: 1146-1159, 2013.

5. Frank DN, St Amand AL, Feldman RA, Boedeker EC, Harpaz N and Pace NR: Molecular-phylogenetic characterization of microbial community imbalances in human inflammatory bowel diseases. Proc Natl Acad Sci USA 104: 13780-13785, 2007.

6. Martinez C, Antolin M, Santos J, Torrejon A, Casellas F, Borruel N, Guarner F and Malagelada JR: Unstable composition of the fecal microbiota in ulcerative colitis during clinical remission. Am J Gastroenterol 103: 643-648, 2008.

7. Morgan XC, Tickle TL, Sokol H, Gevers D, Devaney KL, Ward DV, Reyes JA, Shah SA, LeLeiko N, Snapper SB, et al: Dysfunction of the intestinal microbiome in inflammatory bowel disease and treatment. Genome Biol 13: R79, 2012.

8. Turnbaugh PJ, Ley RE, Mahowald MA, Magrini V, Mardis ER and Gordon JI: An obesity-associated gut microbiome with increased capacity for energy harvest. Nature 444: 1027-1031, 2006.

9. Duncan SH, Lobley GE, Holtrop G, Ince J, Johnstone AM, Louis P and Flint HJ: Human colonic microbiota associated with diet, obesity and weight loss. Int J Obes 32: 1720-1724, 2008.

10. Schwiertz A, Taras D, Schäfer K, Beijer S, Bos NA, Donus C and Hardt PD: Microbiota and SCFA in lean and overweight healthy subjects. Obesity (Silver Spring) 18: 190-195, 2010.

11. Irrazábal T, Belcheva A, Girardin SE, Martin A and Philpott DJ: The multifaceted role of the intestinal microbiota in colon cancer. Mol Cell 54: 309-320, 2014.
12. Schwabe RF and Jobin C: The microbiome and cancer. Nat Rev Cancer 13: 800-812, 2013.

13. Ernst M, Najdovska M, Grail D, Lundgren-May T, Buchert M, Tye H, Matthews VB, Armes J, Bhathal PS, Hughes NR, et al: STAT3 and STAT1 mediate IL-11-dependent and inflammation-associated gastric tumorigenesis in gp130 receptor mutant mice. J Clin Invest 118: 1727-1738, 2008.

14. Wu S, Rhee KJ, Albesiano E, Rabizadeh S, Wu X, Yen HR, Huso DL, Brancati FL, Wick E, McAllister F, et al: A human colonic commensal promotes colon tumorigenesis via activation of T helper type $17 \mathrm{~T}$ cell responses. Nat Med 15: 1016-1022, 2009.

15. Winter SE, Lopez CA and Bäumler AJ: The dynamics of gut-associated microbial communities during inflammation. EMBO Rep 14: 319-327, 2013.

16. Kuper H, Adami HO and Trichopoulos D: Infections as a major preventable cause of human cancer. J Intern Med 248: 171-183, 2000.

17. Mantovani A, Garlanda C and Allavena P: Molecular pathways and targets in cancer-related inflammation. Ann Med 42: 161-170, 2010.

18. Lundberg JO, Weitzberg E, Cole JA and Benjamin N: Nitrate, bacteria and human health. Nat Rev Microbiol 2: 593-602, 2004.

19. Belcheva A, Green B, Weiss A, Streutker C and Martin A: Elevated incidence of polyp formation in APC( $\left.\mathrm{Min} /{ }^{+}\right) \mathrm{Msh} 2^{-/}$ mice is independent of nitric oxide-induced DNA mutations. PLoS One 8: e65204, 2013.

20. Cooke MS, Evans MD, Dizdaroglu M and Lunec J: Oxidative DNA damage: Mechanisms, mutation, and disease. FASEB J 17: 1195-1214, 2003.

21. Evans MD, Dizdaroglu M and Cooke MS: Oxidative DNA damage and disease: Induction, repair and significance. Mutat Res 567: 1-61, 2004.

22. Christl SU, Scheppach W and Kasper H: Hydrogen metabolism in the large intestine - physiology and clinical implications. Z Gastroenterol 33: 408-413, 1995 (In German).

23. Deplancke B, Finster K, Graham WV, Collier CT, Thurmond JE and Gaskins HR: Gastrointestinal and microbial responses to sulfate-supplemented drinking water in mice. Exp Biol Med (Maywood) 228: 424-433, 2003.

24. Hughes R, Cross AJ, Pollock JR and Bingham S: Dose-dependent effect of dietary meat on endogenous colonic N-nitrosation. Carcinogenesis 22: 199-202, 2001.

25. Norat T and Riboli E: Meat consumption and colorectal cancer: A review of epidemiologic evidence. Nutr Rev 59: 37-47, 2001.

26. Hague A, Manning AM, Hanlon KA, Huschtscha LI, Hart D and Paraskeva C: Sodium butyrate induces apoptosis in human colonic tumour cell lines in a p53-independent pathway: Implications for the possible role of dietary fibre in the prevention of large-bowel cancer. Int J Cancer 55: 498-505, 1993.

27. Heerdt BG, Houston MA and Augenlicht LH: Potentiation by specific short-chain fatty acids of differentiation and apoptosis in human colonic carcinoma cell lines. Cancer Res 54: 3288-3293, 1994.

28. Nagashima K, Mochizuki J, Hisada T, Suzuki S and Shimomura K: Phylogenetic analysis of 16 S ribosomal RNA gene sequences from human fecal microbiota and improved utility of terminal restriction fragment length polymorphism profiling. Biosci Microflora 25: 99-107, 2006.

29. Nagashima K, Hisada T, Sato M and Mochizuki J: Application of new primer-enzyme combinations to terminal restriction fragment length polymorphism profiling of bacterial populations in human feces. Appl Environ Microbiol 69: 1251-1262, 2003.

30. Takahashi S, Tomita J, Nishioka K, Hisada T and Nishijima M: Development of a prokaryotic universal primer for simultaneous analysis of Bacteria and Archaea using next-generation sequencing. PLoS One 9: e105592, 2014.

31. Muyzer G, de Waal EC and Uitterlinden AG: Profiling of complex microbial populations by denaturing gradient gel electrophoresis analysis of polymerase chain reaction-amplified genes coding for 16S rRNA. Appl Environ Microbiol 59: 695-700, 1993.

32. Caporaso JG, Lauber CL, Walters WA, Berg-Lyons D, Lozupone CA, Turnbaugh PJ, Fierer N and Knight R: Global patterns of 16S rRNA diversity at a depth of millions of sequences per sample. Proc Natl Acad Sci USA 108 (Suppl 1): 4516-4522, 2011.

33. Castellarin M, Warren RL, Freeman JD, Dreolini L, Krzywinski M, Strauss J, Barnes R, Watson P, Allen-Vercoe E, Moore RA, et al: Fusobacterium nucleatum infection is prevalent in human colorectal carcinoma. Genome Res 22: 299-306, 2012. 
34. Kostic AD, Gevers D, Pedamallu CS, Michaud M, Duke F, Earl AM, Ojesina AI, Jung J, Bass AJ, Tabernero J, et al: Genomic analysis identifies association of Fusobacterium with colorectal carcinoma. Genome Res 22: 292-298, 2012.

35. Kostic AD, Chun E, Robertson L, Glickman JN, Gallini CA, Michaud M, Clancy TE, Chung DC, Lochhead P, Hold GL, et al: Fusobacterium nucleatum potentiates intestinal tumorigenesis and modulates the tumor-immune microenvironment. Cell Host Microbe 14: 207-215, 2013.

36. Rubinstein MR, Wang X, Liu W, Hao Y, Cai G and Han YW: Fusobacterium nucleatum promotes colorectal carcinogenesis by modulating E-cadherin/ $\beta$-catenin signaling via its FadA adhesin. Cell Host Microbe 14: 195-206, 2013.

37. Matthies A, Blaut $M$ and Braune A: Isolation of a human intestinal bacterium capable of daidzein and genistein conversion. Appl Environ Microbiol 75: 1740-1744, 2009.

38. Setchell KD and Clerici C: Equol: History, chemistry, and formation. J Nutr 140: 1355S-1362S, 2010.

39. Watanabe S, Yamaguchi M, Sobue T, Takahashi T, Miura T, Arai Y,Mazur W, Wähälä K and Adlercreutz H: Pharmacokinetics of soybean isoflavones in plasma, urine and feces of men after ingestion of $60 \mathrm{~g}$ baked soybean powder (kinako). J Nutr 128 $1710-1715,1998$

40. Setchell KD and Cole SJ: Method of defining equol-producer status and its frequency among vegetarians. J Nutr 136: 2188-2193, 2006.

41. Setchell KD, Zhao X, Shoaf SE and Ragland K: The pharmacokinetics of S-(-)equol administered as SE5-OH tablets to healthy postmenopausal women. J Nutr 139: 2037-2043, 2009.

42. Song KB, Atkinson C, Frankenfeld CL, Jokela T, Wähälä K, Thomas WK and Lampe JW: Prevalence of daidzein-metabolizing phenotypes differs between Caucasian and Korean American women and girls. J Nutr 136: 1347-1351, 2006.

43. Setchell KD: Phytoestrogens: The biochemistry, physiology, and implications for human health of soy isoflavones. Am J Clin Nutr 68 (Suppl 6): 1333S-1346S, 1998.

44. Cai Y, Guo K, Chen C, Wang P, Zhang B, Zhou Q, Mei F and Su Y: Soya isoflavone consumption in relation to carotid intima-media thickness in Chinese equol excretors aged 40-65 years. Br J Nutr 108: 1698-1704, 2012.

45. Lund TD, Munson DJ, Haldy ME, Setchell KD, Lephart ED and Handa RJ: Equol is a novel anti-androgen that inhibits prostate growth and hormone feedback. Biol Reprod 70: 1188-1195, 2004
46. Brown NM, Belles CA, Lindley SL, Zimmer-Nechemias L, Witte DP, Kim MO and Setchell KD: Mammary gland differentiation by early life exposure to enantiomers of the soy isoflavone metabolite equol. Food Chem Toxicol 48: 3042-3050, 2010.

47. Uematsu H, Sato N, Hossain MZ, Ikeda T and Hoshino E: Degradation of arginine and other amino acids by butyrate-producing asaccharolytic anaerobic Gram-positive rods in periodontal pockets. Arch Oral Biol 48: 423-429, 2003.

48. Barcenilla A, Pryde SE, Martin JC, Duncan SH, Stewart CS, Henderson $C$ and Flint HJ: Phylogenetic relationships of butyrate-producing bacteria from the human gut. Appl Environ Microbiol 66: 1654-1661, 2000.

49. Wong JM, de Souza R, Kendall CW, Emam A and Jenkins DJ: Colonic health: Fermentation and short chain fatty acids. J Clin Gastroenterol 40: 235-243, 2006.

50. Dronamraju SS, Coxhead JM, Kelly SB and Mathers JC: Differential antineoplastic effects of butyrate in cells with and without a functioning DNA mismatch repair. Nutr Cancer 62: 105-115, 2010.

51. Ooi CC, Good NM, Williams DB, Lewanowitsch T, Cosgrove LJ Lockett TJ and Head RJ: Efficacy of butyrate analogues in HT-29 cancer cells. Clin Exp Pharmacol Physiol 37: 482-489, 2010.

52. Roy MJ, Dionne S, Marx G, Qureshi I, Sarma D, Levy E and Seidman EG: In vitro studies on the inhibition of colon cancer by butyrate and carnitine. Nutrition 25: 1193-1201, 2009.

53. Freier TA, Beitz DC, Li L and Hartman PA: Characterization of Eubacterium coprostanoligenes sp. nov., a cholesterol-reducing anaerobe. Int J Syst Bacteriol 44: 137-142, 1994.

54. Stadler J, Stern HS, Yeung KS, McGuire V, Furrer R, Marcon N and Bruce WR: Effect of high fat consumption on cell proliferation activity of colorectal mucosa and on soluble faecal bile acids. Gut 29: 1326-1331, 1988 .

55. Ou J, Carbonero F, Zoetendal EG, DeLany JP, Wang M, Newton K, Gaskins HR and O'Keefe SJ: Diet, microbiota, and microbial metabolites in colon cancer risk in rural Africans and African Americans. Am J Clin Nutr 98: 111-120, 2013.

56. Mantovani A, Allavena P, Sica A and Balkwill F: Cancer-related inflammation. Nature 454: 436-444, 2008.

57. Toprak NU, Yagci A, Gulluoglu BM, Akin ML, Demirkalem P, Celenk T and Soyletir G: A possible role of Bacteroides fragilis enterotoxin in the aetiology of colorectal cancer. Clin Microbiol Infect 12: 782-786, 2006. 